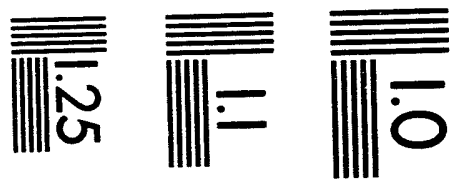

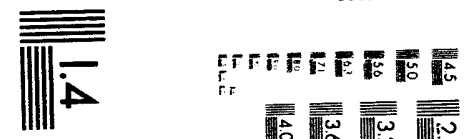

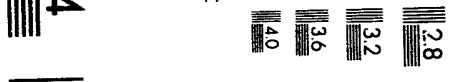

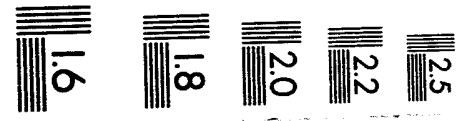



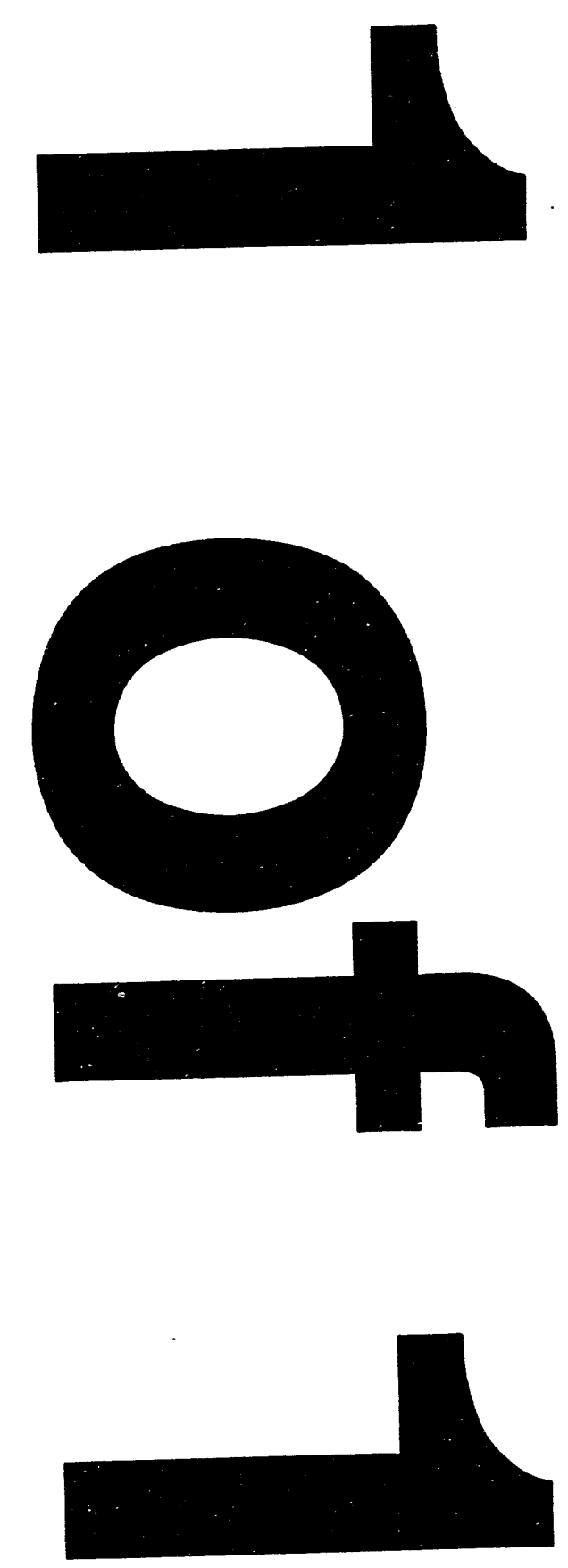


\title{
Wafer-Level Pulsed-DC Electromigration Response at Very High Frequencies
}

\author{
Donald G. Pierce, Eric S. Snyder, Scot E. Swanson and Lloyd W. Irwin \\ Sandia National Laboratories, Electronics Quality/Reliability Center \\ Reliability Physics Department 2276, P.O. Box 5800, Albuquerque, NM 87185
}

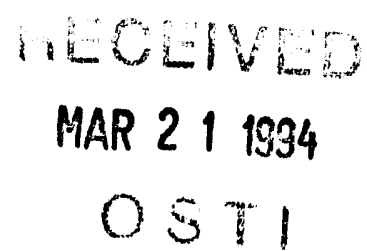

505-845-9375, FAX: 505-844-2991

\begin{abstract}
DC and pulsed-DC electromigration tests were performed at the wafer level using standard and self-stressing test structures. DC characterization tests over a very large temperature range $\left(180\right.$ to $\left.560^{\circ} \mathrm{C}\right)$ were consistent with an interface diffusion mechanism in parallel with lattice diffusion. That data allowed for extraction of the respective activation energies and the diffusion coefficient of the rapid mechanism. The ability to extract simultaneously a defect-based diffusion coefficient and activation energy is significant given the extreme difficulty in making those measurements in aluminum. The pulsed-DC experiments were conducted over a range that includes the highest frequency to date, from $\mathrm{DC}$ to $500 \mathrm{MHz}$. Measurements were also made as a function of duty factor from $15 \%$ to $100 \%$ at selected frequencies. The data shows that the pulsed-DC lifetime is consistent with the average current density model at high (>10 MHz) frequencies and showed no additional effects at the highest frequency tested $(500 \mathrm{MHz})$. At low frequencies, we attribute the lessened enhancement to thermal effects rather than vacancy relaxation effects. Finally, the deviation in lifetime from the expected current density dependence, characterized over $1 \frac{1}{2}$ orders of magnitude in current density, is explained in terms of a shift in the boundary condition for electromigration as the current density is decreased.
\end{abstract}

\section{INTRODUCTION}

The reliability of integrated circuits under pulsed-DC and AC conditions is of great interest to manufacturers and users of those products. This is because most IC structures are subjected to time-varying signals under operating conditions, while reliability characterization is usually done under DC conditions. Of added interest is potential enhancement in the electromigration lifetime at low duty factors during pulsed operation. Enhancement is of practical interest because it allows for less stringent current density design rules for metal lines operating in that mode. The interest in these factors is borne out by the considerable body of experimental and theoretical work on electromigration response under pulsed-DC and $\mathrm{AC}$ conditions, discussed briefly below.

The effects of frequency and duty factor on pulsed-DC electromigration are conveniently discussed using a modified form of Black's Equation [1]

$$
t_{f}=\frac{A}{j^{n} r^{m}} e^{\frac{E_{a}}{k T}}
$$

where $t_{f}$ is the median time-to-failure of the metallization, $A$ is a material and geometry-dependent constant, $j$ is the current density, $n$ is the current density exponent normally assumed to be $2, r$ is the duty factor, $m$ is a constant, $E_{a}$ is the activation energy, $k$ is Boltzmann's constant and $T$ is the temperature. If electromigration depends on frequency we would expect $A$ to be frequency-dependent, as that term contains factors relating to the void nucleation mechanism (e.g., see Iloyd [2]). If the electromigration duty factor dependence is simply explained by damage occurring only during the "on" portion of the current pulse with no annealing of damage during the "off" portion, then $m=1$ in Equation 1; this is sometimes called the "on-time" model. If annealing (or back diffusion) does occur, then $m$ will be greater than 1 , and the pulse response is said to be enhanced. If $m=n$, then Equation 1 yields the average current density model where the time average of the current governs the duty factor response.

Electromigration frequency response measurements reported in the literature have yielded consistent results. The measurements to date have been limited to moderate frequencies ( $<10 \mathrm{MHz}$ ) with the exception of Kwok et al. [3], who extended the range to $200 \mathrm{MHz}$. Unfortunately, their lowest frequency was $50 \mathrm{MHz}$, which makes it difficult to compare that work to other studies. In general, the lifetime is constant with respect to frequency, usually a factor of 2 above DC lifetime for $50 \%$ duty factor, until a transition frequency $\left(f_{0}\right)$ is reached, where it increases to a higher value [4-7]. It is generally assumed that the increase in lifetime occurs when the "on" pulse width during one period falls below the characteristic time constant for the vacancy concentration to reach equilibrium [6]. That increase usually occurs in the range of $1 \mathrm{~Hz}$ to a few $\mathrm{kHz}$. One notable exception to constant lifetime above the transition frequency was observed by Suehle and Schafft [5], who found a decrease in lifetime around $1 \mathrm{MHz}$ with a subsequent increase for higher frequencies. That response seemed to be linked to current density and disappeared as the current density decreased. Unfortunately, their frequency range was limited to $10 \mathrm{MHz}$ so the behavior beyond that point could not be ascertained.

The data on duty factor response has shown far more variation. The early work of Miller [8] generated considerable excitement, as he found an exponential dependence of lifetime with duty factor, with very large enhancements at low duty factors. Experimental work since then [3,5,7,9-14] has not shown such large enhancements, with the value of $m$ usually around 2. Larger values of $m$ (from 3 to 5 ) have also been reported $[3,9,13]$. When $m$ deviates from 2 , it appears to be strongly correlated to the current density exponent, $n$. Based on those results, it is generally assumed that the electromigration

DISTAIBUTION OF THIS DOCUMENT IS UNLIMITED 


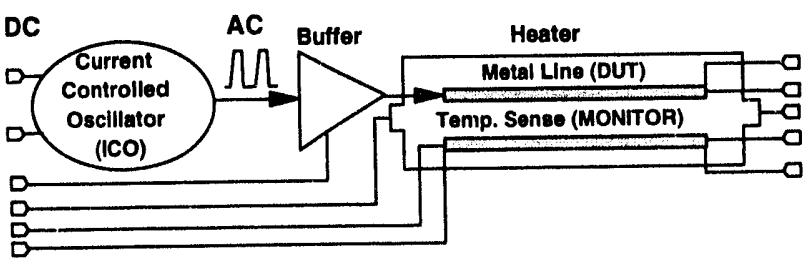

Figure 1. Block diagram of the self-stressing test structure (after [18]).

response under pulsed conditions is a function of the average current density, which results when $m=n$.

The observed duty factor enhancement in electromigration lifetime has led to a variety of models. Most are based upon consideration of the time constant associated with vacancies attaining equilibrium $[8,10,14,15]$. With the exception of [8] all predict $m=2$ for pulse widths less than the vacancy response time. A different approach was taken by Clement [16], who solved the forced diffusion equation numerically with two simple boundary conditions. He also obtained $m=2$. Thus, simple transport theory yields the same result as vacancy relaxation methods.

The experimental work on frequency and duty cycle effects outlined above has fallen short of the frequencies encountered in typical VLSI circuits because of the difficulty and expense of injecting very high frequency signals into packaged structures. To overcome those difficulties, we have developed a selfstressing electromigration test structure with a high-frequency capability limited only by the IC fabrication technology [17,18]. In this paper we report the results of our initial tests using the self-stressing structure at the wafer level. The data spans frequencies from $1 \mathrm{~Hz}$ to $500 \mathrm{MHz}$, with duty factor measurements made at $10 \mathrm{kHz}$ and $200 \mathrm{MHz}$. DC tests were also performed on ASTM standard [19] and the self-stressing test structures in order to characterize the activation energy for diffusion and any additional effects due to current density.

\section{TEST STRUCTURE DESCRIPTION}

Each die location on the wafer contained the DC and selfstressing test structures. The DC (non-self stressing) structures were $1.25 \mu \mathrm{m}$ wide, $7000 \AA$ thick Al-Cu(1\%) alloy deposited on a TiW barrier layer (deposited in a nitrogen ambient). The barrier layer was $1300 \AA$ thick and slightly wider than the Al alloy. The structures were deposited over $1.2 \mu \mathrm{m}$ of oxide and were covered with TEOS interlevel dielectric, glass passivation and a nitride top layer. The TEOS was deposited at $380^{\circ} \mathrm{C}$. The DC structures were $800 \mu \mathrm{m}$ long and conformed to the ASTM standard for electromigration test structures [19].

The metal lines in the self-stressing test structures were somewhat wider than the DC test structures, $1.5 \mu \mathrm{m}$ as opposed to $1.25 \mu \mathrm{m}$. Also, a polysilicon layer was deposited below the self-stressing structures to provide a heat source for electromigration testing. The self-stressing structure also conforms to the ASTM standard with the exception of a pass gate placed in the high sense Kelvin tap. Another ASTMcompliant structure was placed adjacent to the line for the

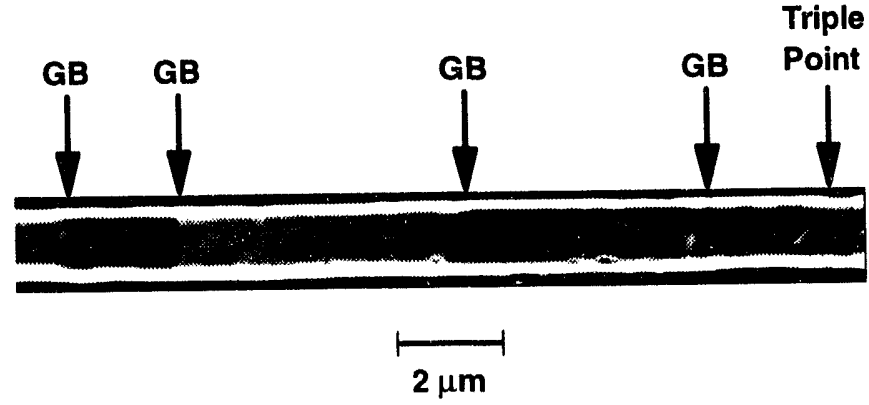

Figure 2. Top view of a typical metal stripe showing near-bamboo grain structure. The image was taken using a focused ion beam.

purpose of thermometry and temperature control. All samples were fabricated on a commercial CMOS line and received the full process.

The self-stressing test structure used is shown schematically in Figure 1. The metal test line (DUT) described above is driven by a current-controlled oscillator/buffer, described in $[17,18]$. The current controlled oscillator/buffer output is set by DC signal levels which control frequency, duty factor, amplitude and polarity of the high frequency waveform. The oscillator/buffer is capable of $\mathrm{AC}$ and pulsed-DC operation, with the maximum frequency limited only by the fabrication technology used, $500 \mathrm{MHz}$ in this instance. The use of a self-stressing structure eliminates the need for external drivers, which reduces the problems of proper impedance matching and its impact on signal fidelity at very high frequencies. The buffer can also be clocked by an off-chip signal generator, which we used for experiments below $1 \mathrm{MHz}$. The oscillator also drives a separate output buffer used to monitor the signal. A polysilicon heater is used to heat the metal stripe instead of a conventional oven. This is an important feature, as raising the entire structure to electromigration temperatures would drastically reduce the output drive and maximum frequency due to reduced mobility in the MOSFETs. Further details of the structure design and operation can be found in [18].

A top view, taken with a focused ion beam, of a representative metal line is shown in Figure 2. In this instance, the line is from a self-stressing structure. As shown by the figure, the grain structure is near-bamboo. That is, the bulk of the grain structure consists of single grains spanning the width of the line with few grain boundary triple points.

\section{EXPERIMENTAL PROCEDURE}

\section{General}

The self-stressing test structure can be used at the wafer level (very high acceleration conditions) or packaged and subjected to more benign stresses. Because this was our first use of the structure, we chose to conduct the experiments at the wafer level, which also removed the need to develop special test boards. That choice also accomplishes the objective of evaluating the applicability of using fast, wafer-level tests to provide initial estimates of frequency and duty factor effects on 


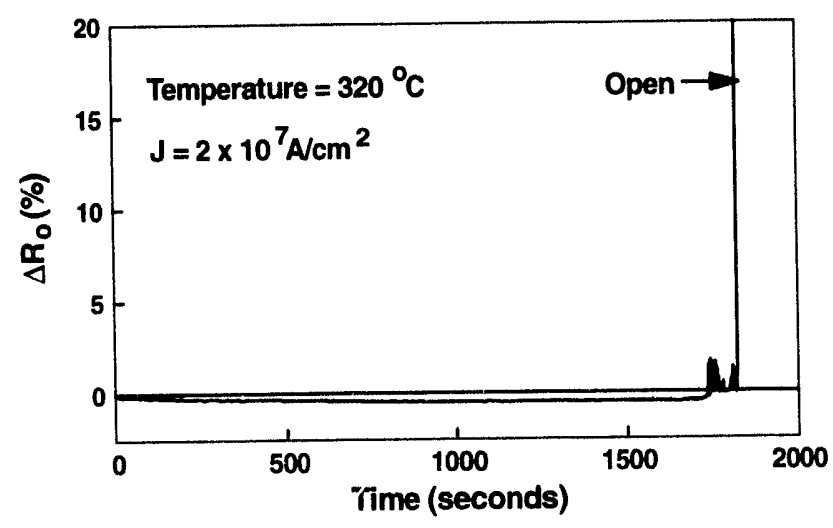

Figure 3. A time profile of resistance change during a typical DC electromigration test shows little sensitivity to the failure criterion chosen.

electromigration times-to-failure. Finally, wafer-level testing allows for access to a much broader range in temperature and current density than is typically available in packaged-structure tests.

Test structures from several wafers from two lots were used in the experiments. Because of possible lot-to-lot and wafer-towafer variations, the temperature coefficients of resistance $(\alpha)$ and thermal resistance $\left(\theta_{\mathrm{sc}}\right)$ were characterized for each structure type and wafer. The temperature coefficient of resistance is key to the thermometry techniques used, and $\theta_{\mathrm{sc}}$ is needed to calculate stripe temperature under pulsed conditions.

Resistance and cross-section measurements showed a $10 \%$ variation in line cross-sectional area between the two lots. Because resistance variations were due to cross-section variations and not material variations, we used the measured resistance to calculate stripe area and hence the applied current density for each stripe. We made no corrections for current flow through the barrier layer since its sheet resistance was estimated as 500 times the sheet resistance of the $\mathrm{Al}-\mathrm{Cu}$ film.

\section{Electromigration}

We performed electromigration tests on the DC structures over very wide temperature $\left(180\right.$ to $560^{\circ} \mathrm{C}$ ) and current density $\left(6 \times 10^{5}\right.$ to $\left.2 \times 10^{7} \mathrm{~A} / \mathrm{cm}^{2}\right)$ ranges in order to bracket the selfstressing test conditions and to get as close to use conditions as was practical. The average sample size was 20 though smaller sample sizes were used at the lower temperatures and current densities because of the large times-to-failure. The bulk of the experiments were "isothermal" electromigration tests at waferlevel, with Joule heating and hence, very high current densities, used to attain the test temperature. The word isothermal is placed in quotes because the temperature is controlled by using the stripe as a thermometer. Since the basal resistance of the stripe will increase as local damage accumulates, hot spots will occur and the temperature of the stripe is in question beyond that point. Also, as damage accumulates, the control algorithm will reduce the applied current to keep the measured resistance constant. Thus, the test would be more aptly named iso-resistive rather

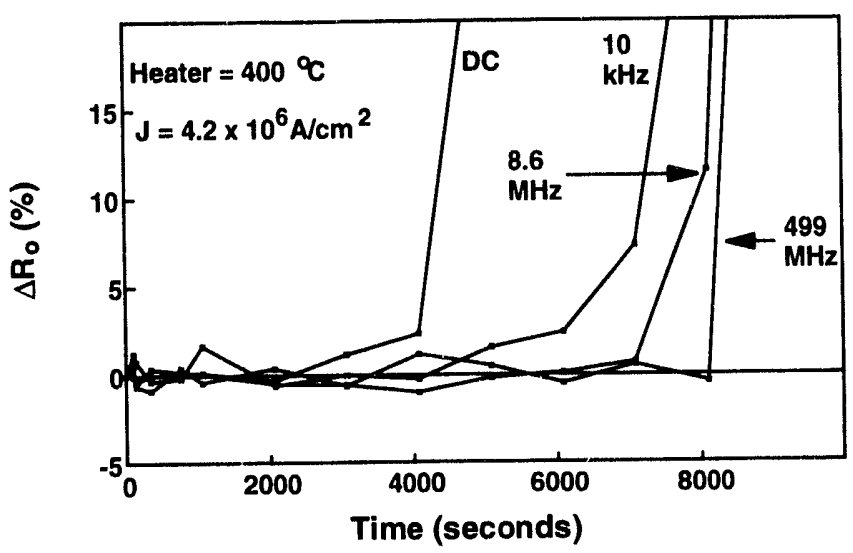

Figure 4. Typical time profiles of resistance changes for self-stressing tests show marked similarity to the "isothermal" profile in Figure 3. A duty 50\% duty factor was used.

than isothermal. While these characteristics would seem to increase test uncertainty with regard to more benign packagedstructure electromigration tests, we have seen excellent correlation to packaged-structure tests (e.g. [20]) for several metallization systems. Further details of the test approach and algorithms used can be found in [21].

In order to investigate the current density effects, DC tests were also done at $420^{\circ} \mathrm{C}$ with the polysilicon heater used to attain part or all of the test temperature. These tests were done either in the "isothermal" mode or the self-stressing constant current mode, depending on the amount of Joule heating desired.

A typical time profile of the change in room temperature stripe resistance $\left(\Delta R_{0}\right)$ for a wafer-level test at $320^{\circ} \mathrm{C}$ is shown in Figure 3. The room temperature resistance is extracted in situ using a thermally derived algorithm [21]. Two important features should be noted from the figure. First, the resistance changes little over most of the test. Thus, in that regime, the test is essentially an isothermal, constant-current test. Second, failure occurs abruptly as an open after a resistance change of little more than $2 \%$. That behavior leads to estimates of the time-to-failure that are insensitive to the failure criterion, which was chosen as a $5 \%$ increase in resistance. That is, the same time-to-failure would have been obtained if the failure criterion was $10 \%, 20 \%$ or an open. All of the tests showed this behavior with resultant opens with the exception of those done at the two lowest temperatures, 180 and $200^{\circ} \mathrm{C}$. For some of the stripes under those conditions the $5 \%$ increase failure criterion was reached before the stripe opened, though onset of failure was still rapid.

\section{Self-Stressing Tests}

The self-stressing tests were done at very high temperatures with the polysilicon heater set to $400^{\circ} \mathrm{C}$. That base temperature, coupled with Joule heating, yielded average stress temperatures ranging from 405 to $420^{\circ} \mathrm{C}$. We chose such extreme temperatures because they yielded times-to-failure short enough 


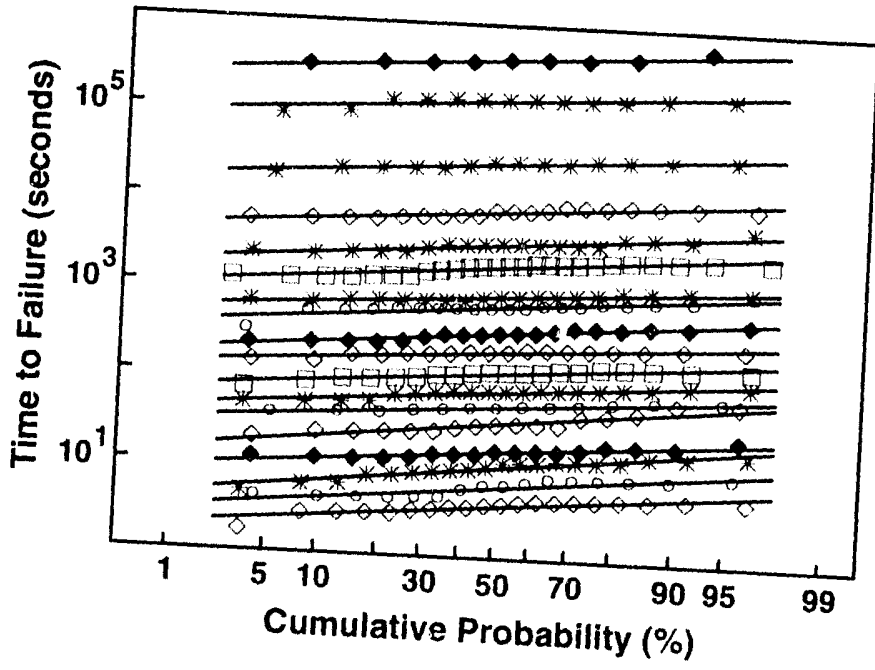

Figure 5. Cumulative time-to-failure distributions for the isothermal tests
at very high current densities. The data is well behaved with failure distributions.

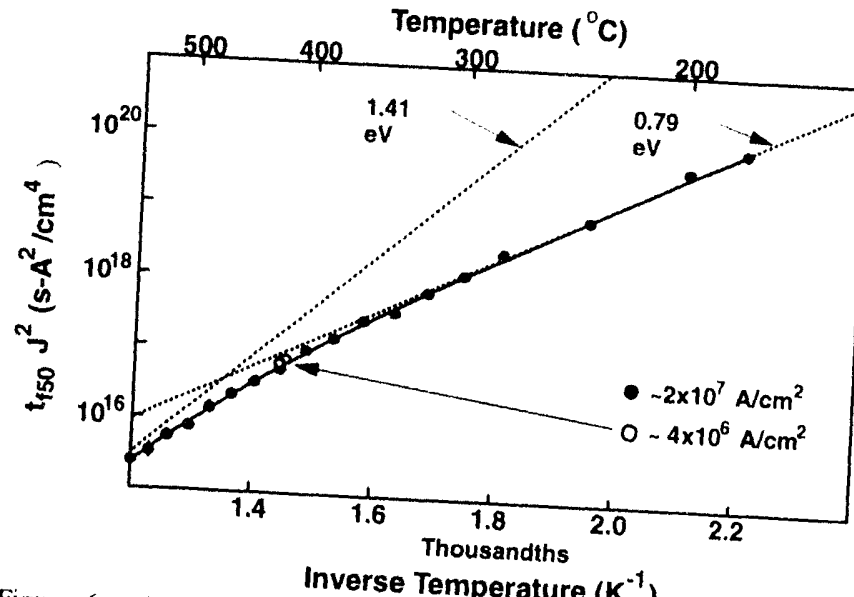

Figure 6. Arrhenius plot of

diffusion mechanisms. Sol of DC electromigration data showing two structures. Open circles are DC test rese DC tests using standard fest structures with the on-hoard buffer.

\section{RESULTS}

(10) and a wide range of feasible with reasonable sample sizes

erency and duty factor conditions.

There are several key differences in the self-stressing tests as compared to the DC wafer-level tests. The self-stressing tests relied on the polysilicon heater to attain the test temperature with modest amounts of Joule heating $\left(5\right.$ to $\left.20^{\circ} \mathrm{C}\right)$. The polysilicon monitor line astively controlled to $\pm 1{ }^{\circ} \mathrm{C}$ through use of the provide parasitic isolation of the hecause of the pass gate used to the bond pad, in situ of the high side of the structure from Instead, at logarithmic intervals, monitoring was not possible. temperature from the DUT and we removed the stress and resistance at room temperature and measured the change in as the logarithmic averature. The failure time was calculated were concerned that of the pre and post failure times. We DUT [22]. stress current showed, several tests conducted with no applied

The self-stressing tests were done at constant applied current whereas the wafer-level DC test algorithm controlled the current to maintain constant stripe resistance. That should have minimal Figure 3 holds; that is, the stipe long as the behavior shown in the bulk of the test. That bethavistance does not change over self-stressing tests, as behavior was also observed for the profile at several pulse frequen by the resistance change time Figure 3. the resistance requencies, shown in Figure 4. Like failure, where a rapid increase constant until the point of resulted in opens.

While the Joule heating was modest in the self-stressing test in temperature, particessary to correct the data for that increase thermal response time. The at pulse widths in the range of the corrections is summarized in Appendix A.

\section{Electromigration Tests}

The results of the DC electromigration characterization test where Joule heating alone was used to attain the test temperature through the data are lognormal least-squares fits. The metal is very well-behaved with unimodal distributions and no evidence 450,000 seconds. The times-to-failure range from 10 to

The data is replotted in Figure 6 in Arrhenius format in order to estimate the activation energy for diffusion in the metal. Note Equation 1 is 2 , which that the current density exponent, 11 , in current densities. The $90 \%$ confirmed by measurements at lower times-to-failure are included forfidence bounds on the median cannot be seen for moded for each data point though they symbols used to which indicates that mote data. The data exhibits curvature over the range, presumably ane diffusion mechanism is active diffusion mechanism. Because laffusion and another, faster, a simple dual diffusion mechanion the data to Appendix B. The solid line in Figure model described in dashed lines denote the contribution 6 is the fit while the mechanism. The fit was contribution from each diffusion with 14 degrees of freedom obtained were $0.79 \mathrm{eV}$ and $1.41 \mathrm{eV}$. The of activation energies range expected for lattice diffusion. The larger value is in the somewhat high for grain bounclary while the smaller value is bamboo structure of the boundary diffusion. Because of the mechanism other than grain botal, we suspect that a diffusion low-temperature diffun grain boundary diffusion is responsible for and the extraction of its diffusion cotal tested. That mechanism, Appendix B. are shown as lognormal probability plots in Figure 5. The line 


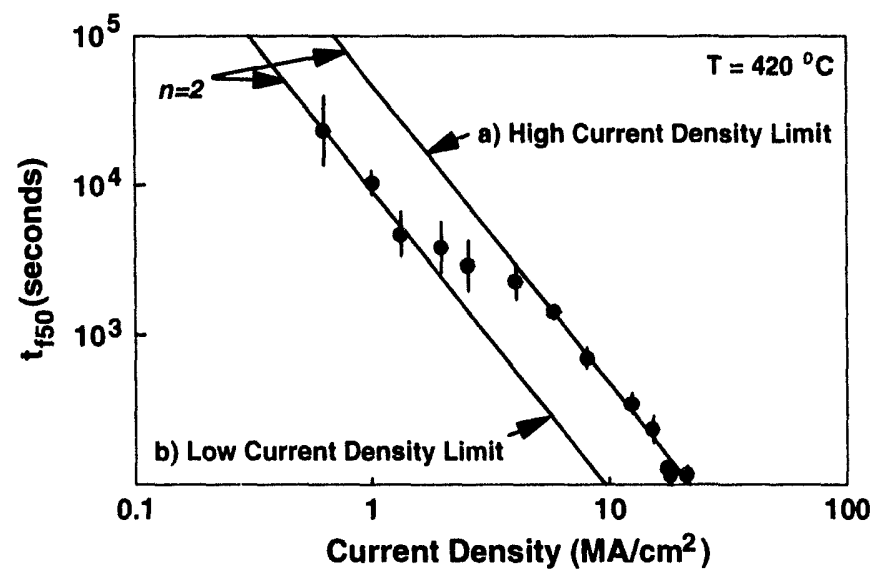

Figure 7. Current density dependence of median times-to-failure at $420{ }^{\circ} \mathrm{C}$. Vertical lines are $90 \%$ confidence bounds.

The curvature in the solid line shown in Figure 6 implies a temperature-dependent activation energy. Because the pulsedDC electromigration tests were done at temperatures (400-420 ${ }^{\circ}$ C) where the electromigration response deviates slightly from low temperature behavior, an effective activation energy, defined in Appendix B, was used to compensate pulsed-DC data for small variations in stripe temperature.

Also shown in Figure 6 are the results of testing the selfstressing structure under DC conditions. The current density used was roughly a factor of 6 less than that used for the "isothermal" test at that point. The agreement with the DC structure test results validates the use of 2 for the current density exponent in the analysis shown in Figure 6.

Suehle and Schafft [6] have pointed out that very highly accelerated wafer-level tests, with commensurate short failure times, may overestimate the time-to-failure. Their arguments are based on vacancy response time considerations where, if the failure time is less than the vacancy response time, then the excess vacancy concentration will be less than the equilibrium value for the stress conditions used. That in turn should lead to an increased time-to-failure at high stress conditions, with a break in the median time-to-failure versus current density characteristic curve. Because our experiments were done under highly accelerated conditions, such an effect could contaminate the results given the wide range in times-to-failure expected.

We characterized the electromigration response as a function of current density to address the concerns outlined above. The experiments were done at current densities ranging from $5 \times 10^{5}$ to $2 \times 10^{7} \mathrm{~A} / \mathrm{cm}^{2}$. The results of that characterization are shown in Figure 7 as a plot of median time-to-failure versus current density. The solid lines in the figure have slopes of -2 . The data clearly shows two regimes with the break between the regimes occurring around $5 \times 10^{6} \mathrm{~A} / \mathrm{cm}^{2}$. This is similar to the behavior observed by Suehle and Schafft, who observed the break around $2 \times 10^{6} \mathrm{~A} / \mathrm{cm}^{2}$.

The remarkable thing about the data shown in Figure 7 is that the current density at the break is close to that observed by Suehle and Schafft [6] yet occurs at a much higher temperature

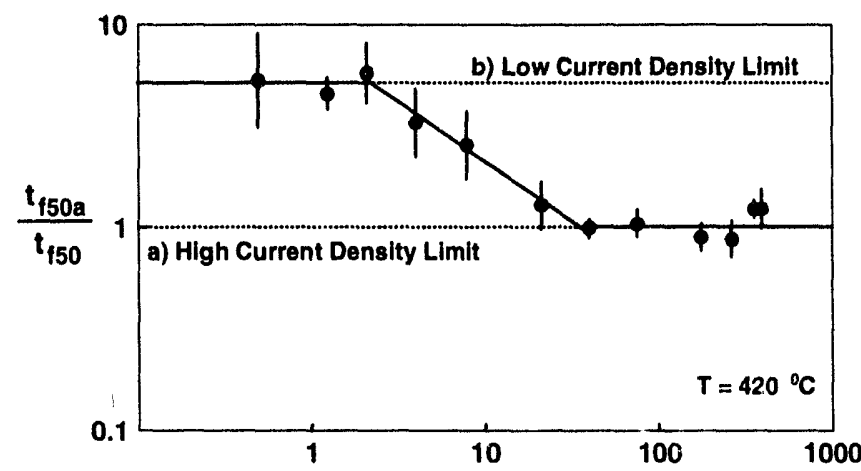

Temperature Rise Above Heater $\left({ }^{\circ} \mathrm{C}\right)$

Figure 8. Correction factor for stripes with peak temperatures that do not produce diffusion barriers at the end of the line. Vertical lines are $90 \%$ confidence bounds.

(420 versus $277^{\circ} \mathrm{C}$ ). If the vacancy response time exhibits an activation energy similar to that of diffusion, then the response time we observed would be on the order of $3 \times 10^{6}$ seconds (830 hours) at $180^{\circ} \mathrm{C}$. That is far outside the range of .01 seconds to 3 hours at $180^{\circ} \mathrm{C}$ cited by Suehle and Schafft based upon their survey of the literature. Such a large value defies physical intuition and we doubt that vacancy response processes are responsible for the behavior shown in Figure 7 and that observed by Suehle and Schafft.

A more straightforward explanation is obtained by noting that the temperature rise above ambient increases as the current density increases. At high stress currents, the temperature drop at the widened ends of the test structure is large and kills the diffusion coefficient in that region. Thus, there is a diffusion barrier at the ends of the line with a zero-flux boundary condition. As the current density is reduced, the temperature gradient-induced barrier becomes less effective until, in the limit of low current densities, it ceases to exist altogether. Thus, the break in Figure 7 simply reflects electromigration under greatly different boundary conditions. Note that if the test structures had deliberate diffusion barriers placed at the ends of the lines then we would expect no break in the response and the data would fall along line a).

Given the model outlined above, we replotted the data in Figure 7 as a function of the increase in stripe temperature above background. The result is shown in Figure 8 where the vertical axis is the line labeled a) in Figure 7 divided by the experimental results. That is, the vertical axis is the "correction factor" needed to bring all of the data shown in Figure 7 into agreement with the high current density trend. We could have normalized Figure 8 to low current densities. However, since the bulk of the DC data was taken with large amounts of Joule heating, normalization to the high current density limit provides for less extrapolation of the data.

The horizontal dashed lines in Figure 8 are the high and low current density trend lines shown in Figure 7. The slanted line is a simple power law fit to the transition region. The decision to use a power law is purely empirical and reflects no underlying 


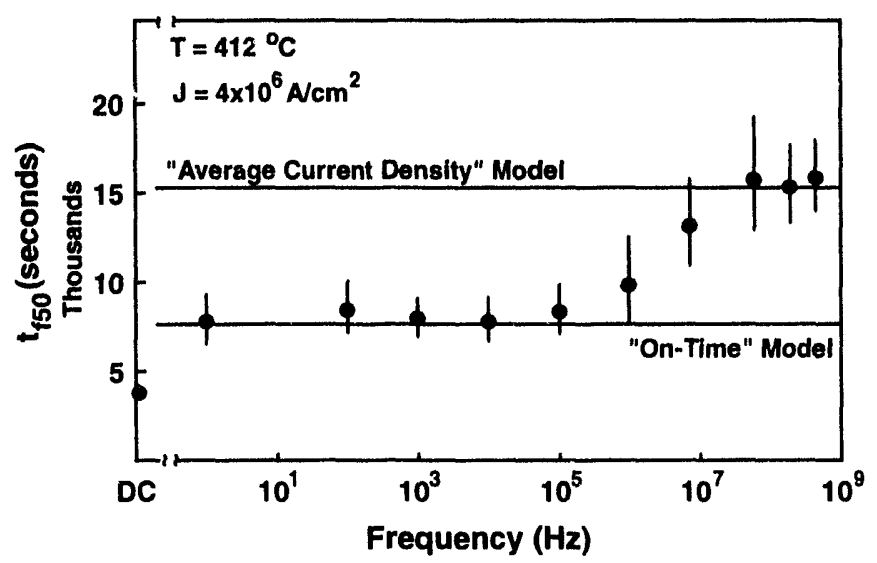

Figure 9. Frequency response of the self-stressing test structures at $50 \%$ duty factor. Vertical lines are $90 \%$ confidence bounds. The increase with frequency is due to thermal effects, rather than characteristic vacancy relaxation times.

physics. The solid line in the figure was used to correct the pulsed-DC data for the effect of changing boundary conditions.

\section{Pulsed-DC Tests}

The median time-to-failure for the self-stressing structures is plotted in Figure 9 versus frequency at $50 \%$ duty factor. The frequencies range from $1 \mathrm{~Hz}$ to $500 \mathrm{MHz}$. The $90 \%$ confidence bounds on the medians are shown as vertical lines. Also shown are two horizontal lines at twice and four times the DC time-tofailure. Those lines represent the predictions of the "on-time" and average current density models for $50 \%$ duty factor and a current density exponent of 2 . The data is tightly clustered about the "on-time" model line for frequencies less than $1 \mathrm{MHz}$ and rises to the average current density model at high frequencies. That dependence is due to the difference in stripe temperature when the pulse is on or off. That is, when there is significant Joule heating the temperature of the stripe in the off-portion of the pulse is so low as compared to the on-portion that back diffusion is not effective in annealing damage. The effect does not occur at high frequencies because the temperature does not change significantly between the on and off portion of the waveform, as discussed in Appendix A. This behavior shows that test techniques that allow significant Joule heating $\left(5^{\circ} \mathrm{C}\right.$ or so) should not be used for pulsed-DC electromigration tests with periods below the structure's thermal time constant. The agreement with the average current density model at high frequencies shows no unusual effects up to $500 \mathrm{MHz}$.

Note that the correction factor shown in Figure 8 played an important role in the data analysis because at high frequencies we were operating in the transition region between the boundary conditions. Without that consideration, the data would show no frequency dependence and would fall along the "on-time" model line. Again, care should be taken when testing structures with no chemical diffusion barriers under conditions with significant Joule heating.

The median time-to-failure for the self-stressing test structures is plotted in Figure 10 versus duty factor for frequencies of $10 \mathrm{kHz}$ and $200 \mathrm{MHz}$. Again, the impact of Joule

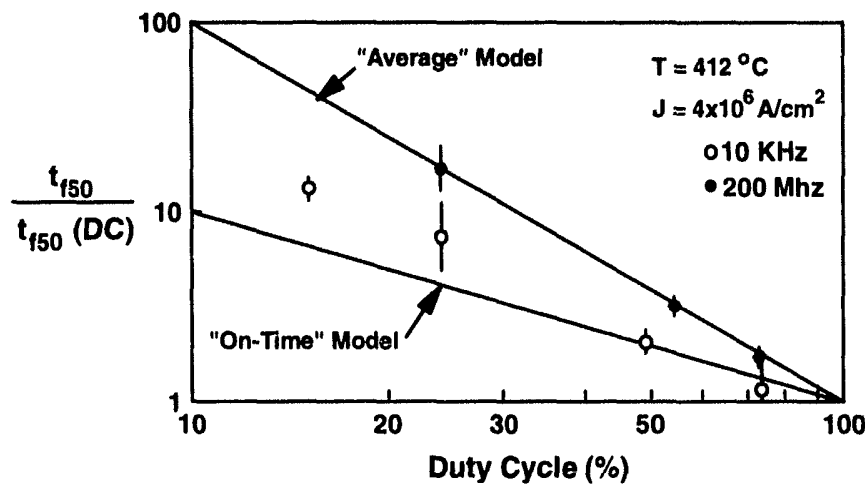

Figure 10. Duty factor response of the median time to failure. The data agrees well with the average current density model at $200 \mathrm{MHz}$. The vertical lines are $90 \%$ confidence bounds.

heating shows up in the low frequency data. While the data does show some enhancement at low duty factors, it is below that expected from the average current density model. The enhancement does show that, with a temperature differential of $20^{\circ} \mathrm{C}$ between on and off states, some back diffusion occurs. In the large Joule heating limit, we would expect the $10 \mathrm{kHz}$ data to follow the "on-time" model.

The data at $200 \mathrm{MHz}$ follows the average current model remarkably well. Thus, experiments with large amounts of Joule heating appear to be appropriate when operating at pulse periods below the thermal time constant. Again note that the correction factors given in Figure 8 played a key role in yielding that agreement. In this instance high current densities are advantageous as they avoid the problem of changing boundary conditions until very low duty factors are reached.

\section{CONCLUSIONS}

We found that the high frequency (up to $500 \mathrm{MHz}$ ) pulsedDC electromigration response of the metal system tested followed the average current density model. At lower frequencies, thermal effects masked the response and yielded smaller amounts of enhancement than expected. Those effects are not important under use conditions unless design rules allow for significant Joule heating.

We also observed the break in the electromigration lifetime dependence on current density observed in [6]. We attribute that break to a change in boundary conditions due to the decrease in the temperature gradient at the ends of the test structure with decreasing current density. That effect would not show up in test structures that incorporate deliberate diffusion barriers at the transition from narrow to wide line width. Knowing the quantitative dependence of lifetime on current density due to that effect proved crucial in proper analysis of the data and highlights the need for vigilance when performing electromigration tests where even modest Joule heating is a factor.

Perhaps the most interesting aspect of the experiments was not the high frequency work but the DC stress test with the analysis described in Appendix B. The electromigration response agreed very well with simple mass transport theory and 


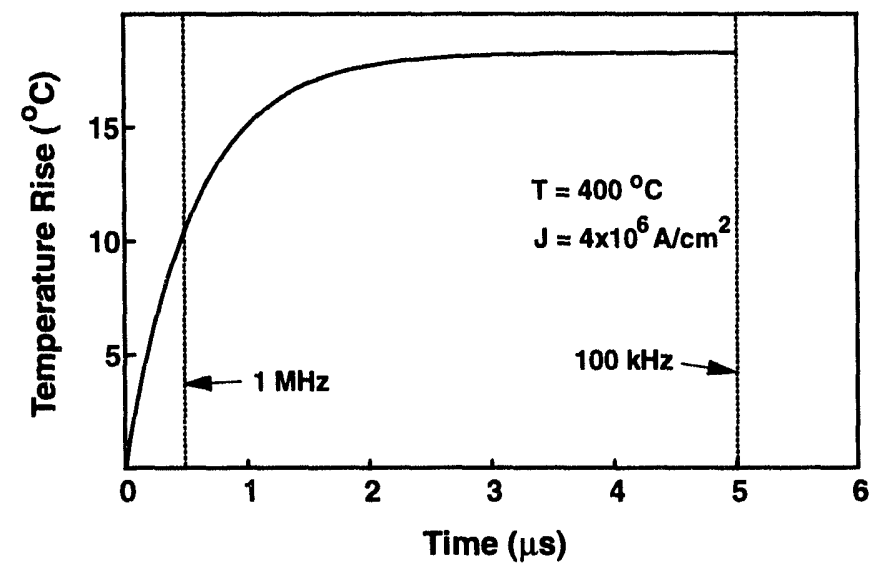

Figure 11. Temperature rise as a function of time for a current step. Note that the temperature comes close to steady state at a few $\mu$ s while adiabatic behavior is apparent at times below a few hundred ns.

allowed us to extract the activation energies for "interface" and lattice diffusion, estimate the "interface" diffusion coefficient and determine the relative impact of the competing diffusion mechanisms as a function of temperature. As electromigration activation energies continue to rise due to process improvements, the relative influence of competing diffusion mechanisms will become more important in packaged-part testing. Wafer-level testing, if done properly, could prove a useful tool in delineating those diffusion mechanisms.

Finally, the self-stressing test structures proved capable of the task of yielding accurate, high quality electromigration data at a wide range of pulse frequencies. As the structures can be used in both the wafer-level (high stress) and packaged-structure (moderate stress) environments, self-stressing test structures should provide a viable alternative to the costly and unwieldy high frequency test systems used for electromigration tests under $\mathrm{AC}$ and pulsed conditions.

\section{ACKNOWLEDGMENTS}

The authors thank Clint Anderson of Philips Semiconductors for fabricating the test structures; Ann Campbell and Jim Rife of Sandia for the grain structure imaging work; Randall Lee of Micrion Corporation for focused ion beam deprocessing; and William Filter of Sandia for his careful and insightful review of the manuscript. This work was sponsored by the United States Department of Energy under prime contract number DE-AC0494AL8500.

\section{APPENDIX A - THERMAL ANALYSIS}

The transient thermal profile for the structures tested was calculated by using the solution to the one-dimensional time dependent heat equation given in [23] and dimensional parameters obtained from the metallization cross-sections. An example of those calculations for a step current input is shown in Figure 11. The vertical lines are reference points to relate the times to frequencies at $50 \%$ duty factor. As expected, adiabatic conditions hold at pulse widths below a few hundred ns, where the temperature will vary slightly about the mean. We expect the pulsed-DC electromigration response to follow that obtained under low stress conditions in the short pulse (high frequency regime). At pulse widths larger than $10 \mathrm{~ms}$, the time to steady state is short compared to the pulse width and the temperature profile closely follows the current pulse shape. In that instance, the off portion of the pulse is at ambient and allows for little diffusion as compared to the on portion of the pulse when Joule heating is significant.

All data was corrected for temperature variations and related to a common temperature $T_{C}$ through the relation:

$$
\left.t_{f c}=\frac{t_{f m}}{t_{p}} e^{\frac{E_{a}}{k T_{c}}} \int_{0}^{t_{p}} K\left(T(t)-T_{h e a t}\right)\right) e^{-\frac{E_{a}}{k T(t)}} d t
$$

where,

$$
T(t)=T_{\text {heat }}+\overline{\Delta T}-\frac{\Delta T_{\text {avg }}}{2}+\Delta T(t)
$$

and $t_{f c}$ is the corrected time-to-failure, $t_{f m}$ is the measured timeto-failure, $t_{p}$ is the pulse width, $E_{a}$ is the effective activation energy (see Appendix B), $\mathrm{K}$ is the boundary condition correction factor given in Figure 8, $T_{\text {heat }}$ is the temperature of the polysilicon heater, $\mathrm{DT}_{\mathrm{avg}}$ is average of the transient profile, $\mathrm{D}$ $T(t)$ is the transient temperature profile shown in Figure 11 and the average temperature increase of the line is [18]

$$
\overline{\Delta T}=\frac{r \theta_{s c} I^{2} R_{0}\left[I+\alpha\left(T_{h e a t}-T_{c}\right)\right]}{1-r \alpha \theta_{s c} I^{2} R_{0}}
$$

where $r$ is the duty factor, $q_{s c}$ is the stripe to chuck thermal resistance (measured), $I$ is the applied current, $T_{C}$ is the chuck temperature, $R_{0}$ is the DUT resistance at $T_{c}$ and $a$ is the temperature coefficient of resistance at $T_{c}$. The data was normalized to the test temperature at $\mathrm{DC}$ conditions, $412^{\circ} \mathrm{C}$, so that frequency and duty factor effects can be directly compared.

\section{APPENDIX B - DIFFUSION CALCULATIONS}

The curvature exhibited by Figure 6 clearly indicates that multiple diffusion mechanisms are active over the temperature range used in the $\mathrm{DC}$ electromigration tests. We modeled that curvature as the result of two parallel diffusion mechanisms. By parallel we mean that all materials are in equilibrium perpendicular to the direction of electromigration-induced transport. That is, there are no concentration gradients transverse to the stripe. That model has the satisfying outcome of yielding lattice transport at high temperatures and a faster transport mechanism at low temperatures. We assume here that interface diffusion is responsible for low temperature diffusion in this metal system. That assumption is discussed in more detail below. 


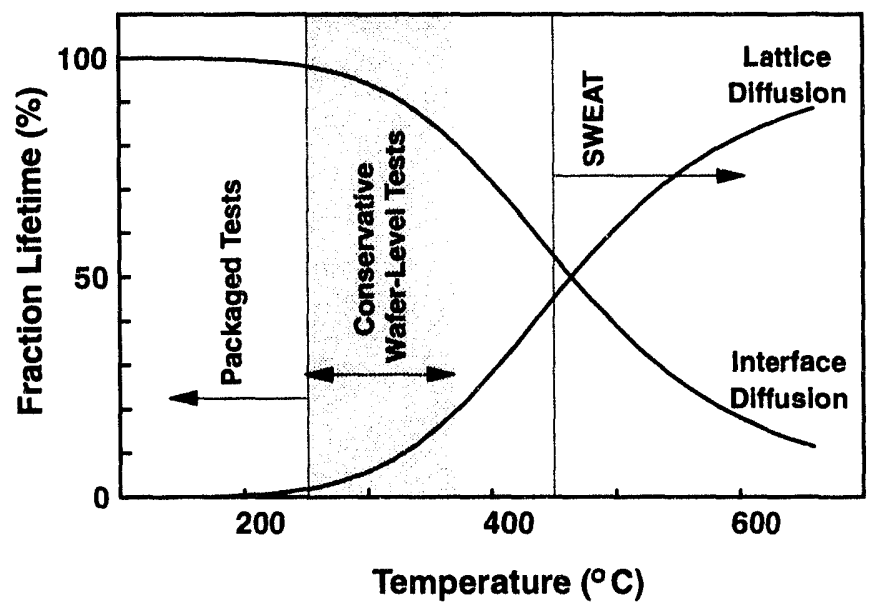

Figure 12. The relative contribution to metal failure due to the two diffusion mechanisms. Note that parameters extracted from the fit in Figure 6 were used to generate the curves.

Based upon the above considerations, the solution to the electromigration equation can be written, by inspection from solutions such as those given by Shatzkes and Lloyd [24], as

$$
\frac{1}{t_{f} j^{2}}=\frac{1}{A_{i}} e^{-\frac{E_{a i}}{k T}}+\frac{1}{A_{l}} e^{-\frac{E_{a l}}{k T}}
$$

where the subscripts denote interface and lattice diffusion respectively. The solid line in Figure 6 is the fit to Equation 2 while the dashed lines denote the contribution from each term in Equation 2. As discussed previously, the fit is excellent.

The form of Equation 2 is similar to that proposed by Dreyer et al. [24]. However, our interpretation is quite different. Equation 2 implies that there is a fast diffusion mechanism always present, regardless of the grain structure of the metal. The model in [24] assumes that grain boundary and lattice diffusion mechanisms appear in series, with the response dictated by the probability density functions of each type of grain configuration. Because of the goodness of fit in Figure 6 and the observed near-bamboo grain structure of the metal, we feel that there is another diffusion mechanism other than grain boundary diffusion operative in parallel with lattice diffusion. That is, if Dreyer's model held for this metal, we would expect to see only lattice diffusion, with no curvature in Figure 6 and a higher activation energy at low temperatures. For lack of a better term, we will call this mechanism "interface diffusion." From a physical standpoint, we suspect that this diffusion mechanism will be active along the $\mathrm{Al}$ - TiW interface. Given the agreement between Equation 2 and the data shown in Figure 6, our assumptions appear warranted.

The result of the fit shown in Figure 6 is presented in somewhat different form in Figure 12. There the fraction of damage caused by each transport mechanism is plotted versus temperature. At temperatures where packaged structure tests are

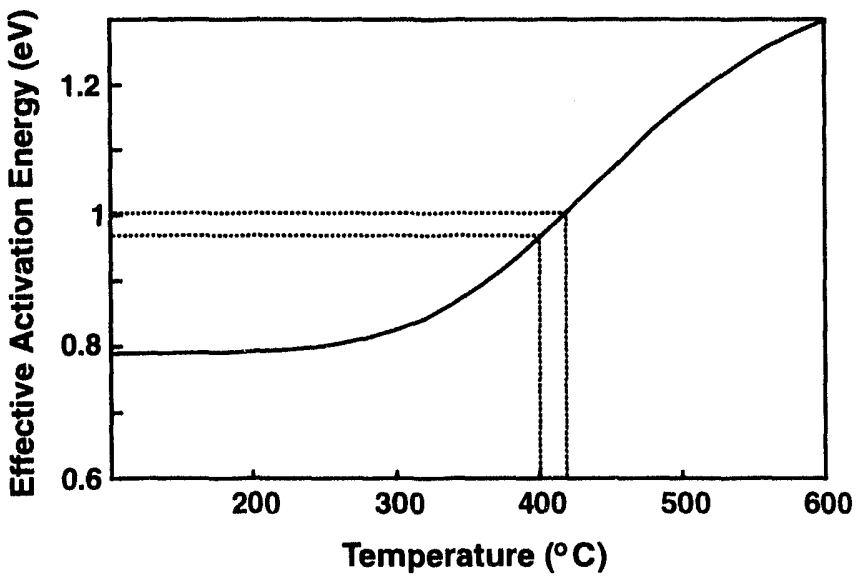

Figure 13. Effective activation energy for the competing diffusion mechanisms versus temperature. The dashed lines denote the temperature range over which the pulsed-DC tests were done.

conducted, the "interface" diffusion mechanism dominates and results can be directly extrapolated to use conditions. At temperatures where conservative wafer-level tests are done, 250 to $350{ }^{\circ} \mathrm{C}$, lattice diffusion will start to affect the results. At temperatures where very fast wafer-level tests such as SWEAT are done $\left(500^{\circ} \mathrm{C}\right)$ lattice diffusion accounts for fully half of the transport and interpretation of results are difficult without a detailed characterization as shown in Figure 6. Characterizations such as these will become more important as grain boundary diffusion is suppressed in advanced metals and hence the impact of multiple diffusion mechanisms is felt at lower temperatures.

For the purposes of data analysis, especially at high temperatures where lattice diffusion plays a role, it is useful to define an effective activation energy [24] based on the tangent to the fitted curve shown in Figure 6 . That result is shown in Figure 13 versus temperature. We used the effective activation energy to correct for small $\left(<20^{\circ} \mathrm{C}\right)$ temperature variations due to Joule heating in the self-stressing tests.

The pre-exponential factors from the fit shown in Figure 6 can be used to estimate the "interface" diffusion coefficient by equating the coefficients to solutions of the electromigration equation [25]

$$
A_{l}=\frac{C_{f}}{D_{0 l}}\left(\frac{k T}{e z_{l}^{*} \rho}\right)^{2}
$$

and

$$
A_{i}=\frac{C_{f}}{\left[\frac{\delta D_{0 i}}{d}\right]}\left(\frac{k T}{e z_{g b}^{*} \rho}\right)^{2}
$$

where $C_{f}$ is the critical vacancy concentration, $\delta$ is the shortcircuit diffusion path thickness, $d$ is the short-circuit diffusion 
path width, $z^{*}$ is the effective charge and all other quantities have been defined. Dividing Equation 4 by Equation 3 yields

$$
\delta D_{0 i}=\frac{A_{l}}{A_{i}}\left(\frac{z_{l}^{*}}{z_{g h}^{*}}\right)^{2} d D_{0 l}
$$

From the fit in Figure $6, A_{i}=1.6 \times 10^{11} \mathrm{~s}-\mathrm{A}^{2} / \mathrm{cm}^{4}$ and $A_{l}=9.3 \times 10^{6} \mathrm{~s}-\mathrm{A}^{2} / \mathrm{cm}^{4}$. Because of the near-bamboo structure of the lines, we set the defect width, $d$, to the linewidth, $1.5 \mu \mathrm{m}$. From the literature, $z_{g b}{ }^{*} \cong 4[26], \quad z l^{*} \cong 26[26]$ and $D_{0 l}=1.71 \mathrm{~cm}^{2} / \mathrm{s}[27]$. We chose to use the value for the effective charge associated with grain boundary diffusion for lack of a better number. The value chosen for $D_{0}$ was associated with an activation energy that was close to the value we measured. Those values used in Equation 5 yield $\delta D_{0 i}=7.5 \times 10^{-7} \mathrm{~cm}^{3} / \mathrm{s}$. The ability to extract simultaneously a defect-based diffusion coefficient and activation energy is significant given the extreme difficulty in making those measurements in aluminum. Generally, the diffusion coefficient is extracted from moving marker experiments while the activation energy is extracted separately from electromigration time-to-failure measurements.

\section{REFERENCES}

[1] J.R. Black, "Mass Transport of Aluminum by Momentum Exchange with Conducting Electrons," Proc. 1967 Ann. Rel. Phys. Symp., pp. 149-151, 1967.

[2] J.R. Lloyd, "Electromigration Failure," J. Appl. Phys., vol. 69, no. 11, pp. 7601-7604, 1991.

[3] T. Kwok, K.K. Chan, H. Chan, and J. Simko, "Thermal StressInduced and Electromigration- Induced Void-Open Failures in $\mathrm{Al}$ and Al-Cu Fine Lines," J. Vac. Sci. Tech., vol. A9, no. 4, pp. 2523-2526, 1991.

[4] J. Tao, N.W. Cheung, and C. Hu, "Electromigration Characteristics of Copper Interconnects," Elect. Dev. Lett., vol. 14, No. 5, pp. 249-251, May 1993.

[5] J.S. Suehle and H.A. Schafft, "Current Density Dependence of Electromigration 150 Enhancement Due to Pulsed Operation," Proc. 28th Ann. Rel. Phys. Symp., pp. 106-110, 1990.

[6] J.S. Suehle and H.A. Schafft, "The Electromigration Damage Response Time and Implicationsfor DC and Pulsed Characterizations," Proc. 27th Ann. Rel. Phys. Symp., pp. 229-233, 1989.

[7] J.A. Maiz, "Characterization of Electromigration under Bidirectional (BC) and Pulsed Unidirectional (PDC) Currents," Proc. 27th Ann. Rel. Phys. Symp., pp. 220-228, 1989.

[8] R.J. Miller, "Electromigration Failure Under Pulse Test Conditions," Proc. 16th Ann. Rel. Phys. Symp. pp. 241-247. 1978.

[9] L.M. Ting, J.S. May, W.R. Hunter and J.W. McPherson, "AC Electromigration Characterization and Modeling of Multilayered Interconnects," Proc. 1993 Ann. Rel. Phys. Symp., pp. 311-316, 1993.
[10] B.K. Liew, N.W. Cheung and C. Hu, "Effects of Self-Heating on Integrated Circuit Metallization Lifetimes," Proc. 1989 IEDM, pp. 323-326, 1989.

[11] K. Hatanaka, T. Noguchi and K. Maeguchi, "A Generalized Lifetime Model for Electromigration under Pulsed-DC/AC Stress Conditions," Proc. Symp. on VLSI Tech., pp. 19-20, 1990.

[12] J.M. Towner and E.P. van der Ven, "Aluminum Electromigration Under Pulsed D.C. Conditions," Proc. 21st Ann. Rel. Phys. Symp., pp. 33-39, 1983.

[13] L. Brooke, "Pulse Current Electromigration Failure Model," Proc. 25th Ann. Rel. Phys. Symp., pp. 136-139, 1987.

[14] C.J. Wu and M.J. McNutt, "Effects of Substrate Thermal Characteristics on Electromigration Behavior of Al Thin Film Conductors," Proc. 21st Ann. Rel. Phys. Symp., pp. 24-31, 1983.

[15] B.K. Liew, N.W. Cheung and C. Hu, "Electromigration Interconnect Failure Under Pulse Test Conditions" Proc. 1988 Symposium on VLSI Technology, pp. 59-60, 1988.

[16] J.J. Clement, "Vacancy Supersaturation Model for Electromigration Failure under DC and Pulsed DC Stress," J. Appl. Phys., vol. 71(9), pp. 4264-4268, May 1992.

[17] E. S. Snyder, D. V. Campbell, S. E. Swanson and D. G. Pierce, "Novel Self-Stressing Test Structures for Realistic High Frequency Reliability Characterization, Proc. 31st Ann. Rel. Phys. Symp., pp. 57-65, 1993.

[18] E.S. Snyder, D.G. Pierce, D.V. Campbell and S.E. Swanson, "Self-Stressing Structures for Electromi zration to $500 \mathrm{MHz}$," 1994 IEEE International Test Structure Conference, March,1994.

[19] "Standard Guide for the Design of Flat, Straight-Line Test Structures for Detecting Metallization Open-Circuit or Resistance-Increase Failure Due to Electromigration, ASTM Standard F1259-89, Annual Book of ASTM Standards, vol. 10.04, pp. 669-670, 1990.

[20] D.G. Pierce and P.G. Brusius, "Correlation Between Moderately and Highly Accelerated Electromigration Tests," Elect. Dev. Lett., vol. 14, no. 6, June 1993.

[21] "SWORD (Sandia Wafer-Level Reliability Software) User's Manual. Hewlett-Packard Co., No. HP94819QU, August 1993.

[22] A.C. Macpherson, W.H. Weisenberger, H.M. Day, and A. Christou, "Effects of Fast Temperature Cycling on Aluminum and Gold Metal Systems," Proc. 13th Rel. Phys. Symp., pp. 113-120, 1975.

[23] D.G. Pierce, "Modelling Metallization Burnout of Integrated Circuits," Proc. 1982 Electrical Overstress/Electrostatic Discharge Symposium, EOS-4, pp. 56-61, September 1982.

[24] M.L. Dreyer, K.Y. Fu, and C.J. Varker, "The Effects of Temperature and Microstructure on the Components of Electromigration Mass Transport," Proc. 1993 Ann. Rel. Phys. Symp., pp. 304-310, 1993.

[25] M. Shatzkes and J.R. Lloyd, "A Model for Conductor Failure Considering Diffusion Concurrently with Electromigration Resulting in a Current Exponent of 2," J. Appl. Phys., vol. 59. no. 11, pp. 3890-3892, 1986.

[26] K.N. Tu, "Electromigration in Stressed Thin Films," Phys Rev. B , vol. 45, pp. 1409-1413, 1992.

[27] T.S. Lundy and J.F. Murdock, "Diffusion of $\mathrm{Al}^{26}$ and $\mathrm{Mn}^{51}$ in Aluminum," J. App. Phys., vol. 33, no. 5, pp. 1671-1673, 1962. 


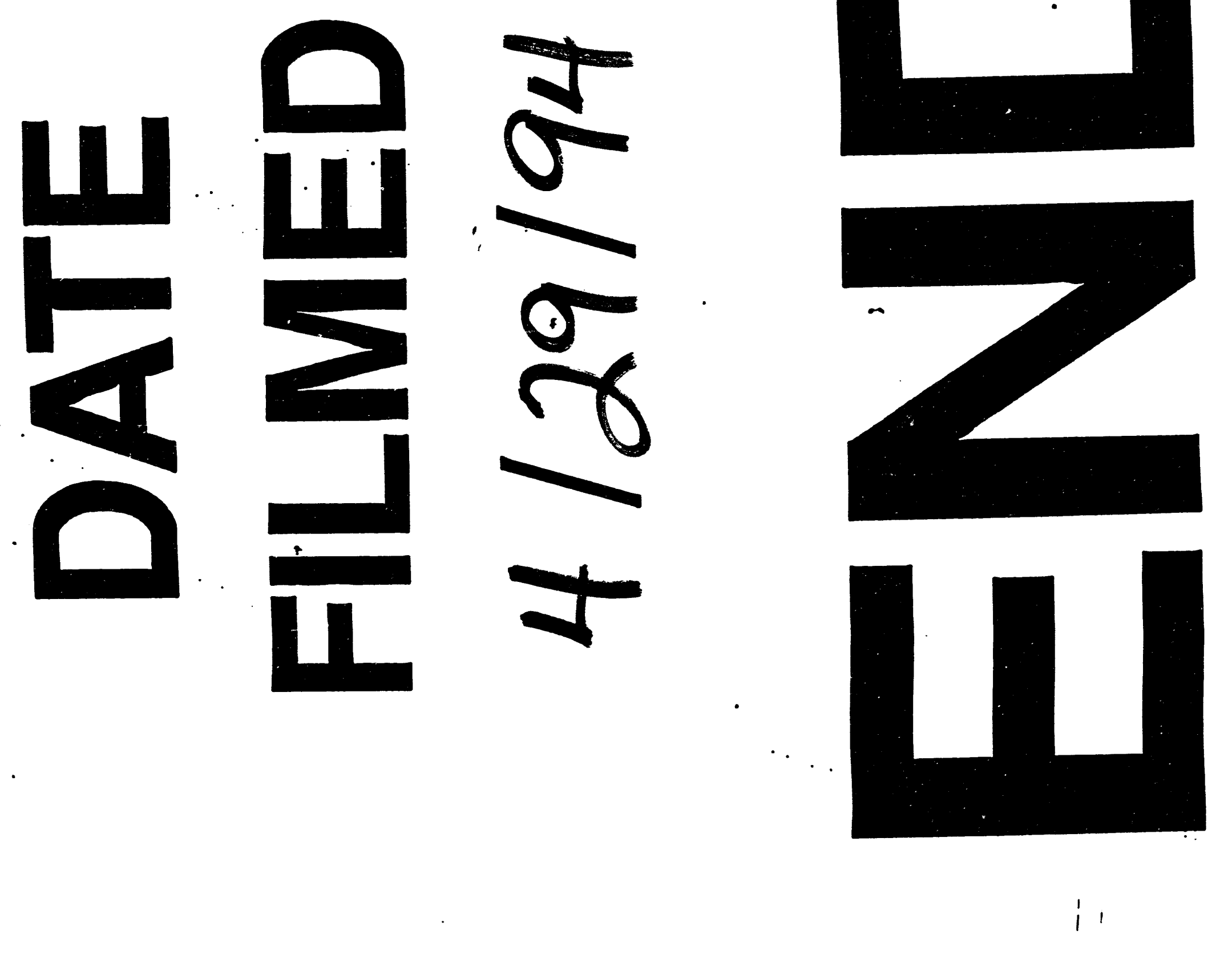


